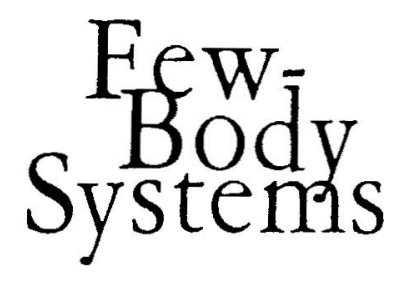

Supplement 10

Few-Body Problems

in Physics '98

Proceedings of the 16th European Conference on Few-Body Problems in Physics,

Autrans, France, June 1-6, 1998

Edited by

B. Desplanques, K. Protasov, B. Silvestre-Brac, J. Carbonell

SpringerWienNewYork 
Dr. Bertrand Desplanques

Dr. Konstantin Protasov

Dr. Bernard Silvestre-Brac

Dr: Jaume Carbonell

Institut des Sciences Nucléaires

Grenoble, France

Printing was supported by Bundesministerium für Wissenschaft und Verkehr, Wien

This work is subject to copyright.

All rights are reserved, whether the whole or part of the material is concemed, specifically those of translation, reprinting, re-use of illustrations, broadcasting, reproduction by photocopying machines or similar means, and storage in data banks.

(C) 1999 Springer-Verlag/Wien

Printed in Austria

Typesetting: Camera-ready by authors

Printing: Druckerei Novographic, A-1238 Wien

Binding: Papyrus, A-1100 Wien

Printed on acid-free and chlorine-free bleached paper

SPIN: 10690263

With 217 Figures and 1 Frontispiece 


\title{
Jastrow-Correlated Configuration-Interaction Description of Light Nuclei
}

\author{
M. Portesi ${ }^{1}$, J. Navarro ${ }^{1}$, R. Guardiola ${ }^{2}$, I. Moliner ${ }^{2}$, R.F. Bishop ${ }^{3}$, \\ N.R. Walet ${ }^{3}$
}

${ }^{1}$ IFIC (Centre Mixt CSIC - Universitat de València), Avda. Dr. Moliner 50, E-46100 Burjassot, Spain

${ }^{2}$ Dept. de Física Atómica, Molecular i Nuclear, Universitat de València, Avda. Dr. Moliner 50, E-46100 Burjassot, Spain

${ }^{3}$ Department of Physics, UMIST, P.O. Box 88, Manchester M60 1QD, U.K.

\begin{abstract}
This work describes recent progress of the UMIST-VALENCIA collaboration on the $a b$ initio study of ground states of light nuclei using realistic forces. The method presented here constructs trial variational wave functions by superimposing a central Jastrow correlation on a state-dependent translationally invariant linearly correlated state, with very promising results.
\end{abstract}

When dealing with finite systems of mutually interacting particles, one of the important difficulties found is the proper treatment of the centre-of-mass (CM) motion. One of the techniques to deal with this problem is the translationally invariant configuration-interaction (TICI) method [1]. This is a variational methodology inspired by a linear version of the coupled-cluster method (CCM), and it differs from the conventional CI formalism in the means of selecting the interacting configurations. The method has mainly been used in a two-body implementation (TICI2), where all correlations are truncated at a two-body level; the interacting configurations correspond to the most general single pair excitations which leave the CM unaffected. This kind of configuration is very similar to those considered in the so-called potential basis in the hyperspherical harmonics formalism. Recently [2, 3], TICI2 has been applied to saturated nuclei within the $0 p$ shell, using state-dependent (SD) correlations between nucleons that interact via a realistic or semirealistic force. The method has also been extended for bosonic systems [4] to the so-called TICC2 approach, which is the full second-order CCM with translational invariance and contains the excitation of an arbitrary number of independent pairs. In the case of ${ }^{4} \mathrm{He}$ there is not a significant improvement when going from TICI2 to TICC2, indicating 
the lack of importance for this nucleus of two-pair excitations. In this work we report on an attempt to go beyond our previous TICI2 calculations. A study of the ${ }^{4} \mathrm{He}$ ground state (g.s.) with tensor forces shows that in order to get a good description we need to mix the operatorial structure of the TICI2 wavefunction with a scalar Jastrow-like factor. We hope to use this methodology as a means of shedding light on how to improve the prediction of the g.s. energy of few-body systems.

In the two-body approximation of CCM, the g.s. wavefunction for an $N$ particle system is written as $\exp \left(S_{1}+S_{2}\right)|\Phi\rangle$, where $|\Phi\rangle$ is an appropriate independent-particle state, and the cluster operators $S_{n}$ promote $n$ particles out of it. The uncorrelated reference state is taken to be a Slater determinant of single-particle harmonic oscillators (HO), and the CM dependence is thereby factorised exactly. To preserve this translational invariance, the excitations produced by $S_{1}$ and $S_{2}$ can not be independent; instead, one has to construct a new operator, $S_{(1,2)}$, which mixes one- and two-body correlations properly. The TICC2 method is based on an expansion of the exponentiated form of $S_{(1,2)}$, at the same time imposing that triplets or higher excitations do not appear; the g.s. energy is obtained as the expectation value of the intrinsic Hamiltonian. TICI2 functions similarly, but one keeps only the linear term in the expansion of $\exp \left(S_{(1,2)}\right)$. In coordinate representation, the TICI2 wavefunction reads

$$
\Psi\left(\boldsymbol{r}_{1}, \ldots, \boldsymbol{r}_{N}\right)=\sum_{i<j} f\left(r_{i j}\right) \Phi\left(\boldsymbol{r}_{1}, \ldots, \boldsymbol{r}_{N}\right)
$$

In this form, it is quite straightforward to introduce state dependence into the pair correlation function by expanding it in an appropriate operatorial basis, $f\left(r_{i j}\right)=\sum_{p} f_{p}\left(r_{i j}\right) \Theta_{p}(i j)$. The basis is generally chosen to be consistent with that of the internucleon potential. For interactions with V4 structure the operators used are $\left\{\Theta_{p}\right\}=\left\{\mathbb{1}, P_{\sigma}, P_{\tau}, P_{\sigma} P_{\tau}\right\}$, with $P_{\sigma}$ and $P_{\tau}$ being the usual spinand isospin-exchange operators, respectively, and for V6-like interactions, the basis also includes the tensor operators $\mathcal{S}_{i j}$ and $\mathcal{S}_{i j} P_{\tau}$ (we note that for ${ }^{4} \mathrm{He}$ the number of relevant operators is actually reduced due to spatial symmetry). The correlation functions $f_{p}$ are taken as combinations of Gaussians, a common technique which gives a very accurate description [1]. Results obtained for ${ }^{4} \mathrm{He}$ [3] show a strong dependence on the shape of the interaction, specially for V6 forces, from what one can conclude that the method suffers a lack of correlations when dealing with realistic potentials.

It is known that Jastrow correlations are very well suited to treat the effects of strong short-range repulsions. This motivated us to introduce into the previous ansatz a scalar Jastrow factor. Simultaneously, we maintain the linear operatorial structure of the TICI2 state, leading to the following J-TICI2 wavefunction:

$$
\Psi\left(\boldsymbol{r}_{1}, \ldots, \boldsymbol{r}_{N}\right)=\prod_{i<j} g\left(r_{i j}\right) \sum_{k<l} \sum_{p} f_{p}\left(r_{k l}\right) \Theta_{p}(k l) \Phi\left(\boldsymbol{r}_{1}, \ldots, \boldsymbol{r}_{N}\right) .
$$

For the sake of simplicity, we use a single Gaussian for the Jastrow factor, $g(r)=1+a \mathrm{e}^{-b r^{2}}$. The trial wavefunction depends on the amplitude $a$ and depth $b$ of the Jastrow function, the $\mathrm{HO}$ length and the functions $f_{p}$. We inust perform 
a variational search of the parameters to minimize $\langle\Psi|H| \Psi\rangle /\langle\Psi \mid \Psi\rangle-E_{\mathrm{CM}}$, where $E_{\mathrm{CM}}$ is the energy related to the CM motion.

We have computed the g.s. energy of the ${ }^{4} \mathrm{He}$ nucleus using several interactions with V4 and V6 structure, the latter coming from truncating various realistic forces to their V6 parts. The calculated energies are given in Tables 1 and 2 , respectively, together with a comparison with other methods.

Table 1. Comparison between different results for the ${ }^{4} \mathrm{He}$ g.s. energy (in $\mathrm{MeV}$ ) using several V4 potentials. S3w is the Wigner part of the S3 potential.

\begin{tabular}{|l|cccc|}
\hline Potential & TICI2 [2] & J-TICI2 & SD-Jastrow [5] & DMC [6] \\
\hline B1 & -37.86 & -38.28 & $-38.27[6]$ & $-38.32 \pm 0.01$ \\
S3 & -28.19 & -30.16 & -29.94 & \\
S3W & -25.41 & -27.20 & -27.21 & $-27.35 \pm 0.02$ \\
MS3 & -27.99 & -29.97 & -29.70 & \\
MT V & -29.45 & -31.21 & -30.88 & $-31.32 \pm 0.02$ \\
MT I/III & -30.81 & -32.70 & -32.01 & \\
\hline
\end{tabular}

Table 2. Same as Table 1, but using the V6 part of several realistic potentials.

\begin{tabular}{|l|cccc|}
\hline Potential & TICI2 [3] & J-TICI2 & VMC [3] & GFMC [7] \\
\hline GPDT & -27.37 & -27.58 & $-27.71 \pm 0.06$ & \\
SSC & -24.12 & -26.74 & $-29.20 \pm 0.12$ & \\
AV14 & -14.77 & -20.37 & $-23.24 \pm 0.08$ & $-24.79 \pm 0.20$ \\
AV18 & -15.40 & -21.08 & $-24.80 \pm 0.09$ & \\
REID & -5.67 & -22.70 & $-27.82 \pm 0.12$ & $-28.30 \pm 0.12$ \\
\hline
\end{tabular}

In Table 1, we compare our J-TICI2 results with previous TICI2 ones, and with a recent calculation using SD-Jastrow correlations parametrised by two Gaussians for each spin-isospin channel; for Wigner-like interactions, we also quote diffusion Monte Carlo (DMC) results. The main difference between J-TICI2 and SD-Jastrow lies in the way of describing the state dependence of the correlation: in the former, the Jastrow factor has a very simple scalar form and all the operatorial dependence is contained in the linear terms; in the latter, the correlation factor has the same operatorial structure as the potential (the product of correlated pairs must be symmetrised to maintain the Fermi statistics). It can be seen that: (i) the inclusion of the Jastrow factor lowers the energy of the pure TICI2 case by $\sim 2 \mathrm{MeV}$ for most interactions; (ii) the energies obtained for the Wigner potentials B1 and S3w from the Jastrowtimes-linear and only-Jastrow correlations practically coincide, but for more realistic interactions with full V4 structure the mixed ansatz works better; (iii) for the MT V potential the one-Gaussian J-TICI2 value is lower than the two-Gaussian Jastrow result (this improvement can be explained by realizing that the MT forces contain a very short-range Yukawa repulsion and thus the Jastrow correlation centres in this region, treating poorly the longer distances); (iv) the proximity of the J-TICI2 and DMC results allows us to conclude that, 
for V4 interactions, the present approach gives a good way to treat the ${ }^{4} \mathrm{He}$ g.s.

In Table 2, in addition to the TICI2 and J-TICI2 descriptions, we include calculations with a V6 SD-Jastrow correlation computed with the variational Monte Carlo (VMC) method, and also the V6 contribution to the energy computed with the Green function Monte Carlo (GFMC) method. The pattern of results is now rather different. The TICI2 results are not good at all for strongly repulsive realistic forces; the effect of including the Jastrow correlation is impressive in some cases, like the Reid one with a gain in binding energy of $17 \mathrm{MeV}$. Nevertheless, with the exception of the very soft GPDT potential, we are still rather far from the $\mathrm{MC}$ results. The hybrid treatment seems however to be a promising method to deal with realistic forces.

We have analysed the optimal values of the variational parameters of the J-TICI2 wavefunction. The HO length does not change very much for different interactions, ranging between 0.71 and $0.78 \mathrm{fm}^{-1}$, and thus gives similar overall sizes for the system. By contrast, the Jastrow parameters present a wider range of variation, mainly due to the different short-range behaviour of the potentials.

In these model calculations we tried to ascertain the importance of superimposing a Jastrow correlation on linear SD pair correlations. The results appear to indicate that this might be a rather good way of describing light systems with realistic interactions. We still obtain several $\mathrm{MeV}$ less binding than the much more precise $\mathrm{MC}$ calculations; our belief is that the central part of the correlation used here is too simple and definite conclusions must be delayed until we are able to deal with a more complex scalar Jastrow correlation. Finally, we note the conceptual similarity between our approach and the correlated basis function (CBF) description of many-body systems.

Acknowledgement. This work is supported by the EEC network No. ERBCHRXCT940456, DGICyT (Spain) contract No. PB92-0820, and EPSRC (UK) research grant GR/L22331.

\section{References}

1. R.F. Bishop et al.: Phys. Rev. C42, 1341 (1990); J. Phys. G16, L61 (1990); Cond. Matt. Theo. 5, p. 253, ed. V.C. Aguilera-Navarro. New York: Plenum Press 1990

R.F. Bishop et al.: Cond. Matt. Theo. 6, p. 405, eds. S. Fantoni and S. Rosati New York: Plenum Press 1991; J. Phys. G17, 857 (1991); ibid. 18, 1157 (1992); ibid. 19, 1163 (1993)

2. R. Guardiola et al.: Nucl. Phys. A609, 218 (1996)

3. I. Moliner et al.: Cond. Matt. Theo. 13, eds. J. da Providencia and R.F. Bishop. New York: Nova Science 1998, in press

4. R. Guardiola et al.: Nucl. Phys. A628, 187 (1998)

5. R. Guardiola and M. Portesi: J. Phys. G24, L37 (1998)

6. R.F. Bishop et al.: J. Phys. G18, L21 (1992)

7. J. Carlson: Phys. Rev. C36, 2026 (1987); ibid. 38, 1879 (1988) 